$\Rightarrow$ METABOLISM AND CANCER

\title{
A sugar rush of DNA methylation
}

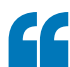

high levels

interfere

with DNA

demethylation

by TET 2

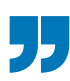

of glucose

Diabetes predisposes to cancer development, but the molecular mechanisms underlying this tumour-promoting effect are poorly understood. Wu et al. now show that elevated glucose levels, which are a hallmark of diabetes, are directly linked to decreased DNA hydroxymethylation, which affects gene expression and contributes to cancer pathology.

DNA cytosine methylation $(5 \mathrm{mC})$ is an epigenetic mark that silences gene expression. This mark can be removed by demethylation, the first step of which involves oxidation of $5 \mathrm{mC}$ to hydroxymethylcytosine (5hmC) by the ten-eleven translocation (TET) family of dioxygenases. Notably, both $5 \mathrm{mC}$ and $5 \mathrm{hmC}$ signatures are frequently deregulated in cancer. Analysis of peripheral blood mononuclear cells (PBMCs) derived from healthy and diabetic individuals revealed that diabetes is associated with a decrease in $5 \mathrm{hmC}$ levels. Experiments using PBMCs and other human cell lines confirmed that high glucose conditions are sufficient to reduce global $5 \mathrm{hmC}$ levels. They also

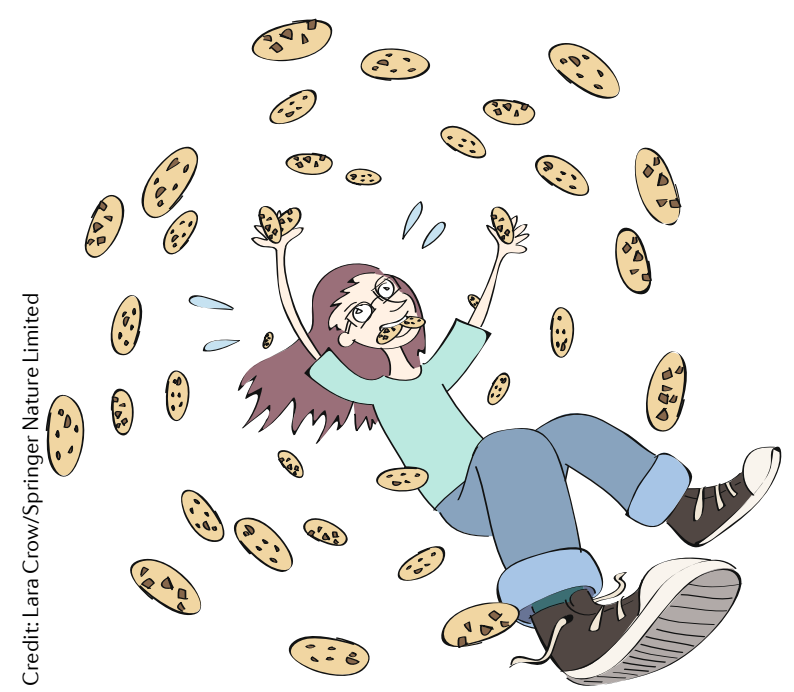

revealed the concomitant decrease of TET2 protein, indicating that high glucose downregulates TET2 protein levels, leading to reduced $5 \mathrm{hmC}$ levels on DNA.

Next, the authors analysed genomic regions that are differentially 5-hydroxymethylated between high and low glucose conditions in a TET2-dependent manner. They used A2058 melanoma cells which normally have low TET2 and $5 \mathrm{hmC}$ levels - in which TET2 was ectopically expressed. TET2 expression was sufficient to raise global $5 \mathrm{hmC}$ levels when cells were cultured under normal glucose, and this effect was compromised in high glucose conditions. They identified $>30,000$ DNA regions that were differentially 5-hydroxymethylated, the majority of which showed a decrease in $5 \mathrm{hmC}$ in high glucose conditions. The majority of the identified regions were localized within genes, and gene ontology analysis revealed strong association of these genes with cancerrelated pathways. Accordingly, several tumour suppressors were downregulated and several tumourpromoting genes were upregulated when TET2-expressing cells were cultured in high glucose conditions. This indicates that high levels of glucose interfere with DNA demethylation by TET2, which has an impact on the expression of cancer-associated genes.

AMP-activated kinase (AMPK) is a nutrient and energy sensor that is potently activated by low glucose and inactivated by high glucose. AMPK phosphorylated TET2 on Ser99 in vitro as well as in TET2-expressing A2058 cells, where TET2 Ser99 phosphorylation was inversely correlated with glucose concentration in culture media. Ser99 phosphorylation is known to protect TET2 from degradation. Accordingly, activation of AMPK by the anti-diabetic drug metformin in TET2-expressing A2058 cells or ectopic expression of a phosphomimetic TET2 mutant augmented the half-life of TET2 and increased $5 \mathrm{hmC}$ levels in high glucose conditions. By contrast, depletion of the nuclear isoform of AMPK decreased the levels of wild-type TET2, but had no effect on a non-phosphorylatable TET2 mutant. These findings indicate that the low levels of $5 \mathrm{hmC}$ seen in high glucose conditions are a consequence of the inhibition of AMPK-mediated TET2 phosphorylation and increased TET2 degradation.

A2058 cells ectopically expressing TET2 showed reduced capacity for anchorage-independent growth (a hallmark of cancer cells) under normal glucose, which was further reduced in the presence of metformin. Furthermore, high glucose concentrations promoted proliferation of these cells, and this effect was abrogated by metformin. Importantly, TET2-expressing A2058 cells formed larger tumours when transplanted into diabetic mice compared with healthy animals. Treatment with metformin reduced tumour growth and this was associated with increased $5 \mathrm{hmC}$ levels in tumour cells.

In summary, this study reveals that an AMPK-TET2-5hmC axis limits tumour growth, and that this axis is impaired in hyperglycaemic conditions owing to glucose-mediated AMPK inhibition. It uncovers a molecular link between diabetes and cancer, which has potential implications for cancer therapy.

Paulina Strzyz

ORIGINAL ARTICLE Wu, D. et al. Glucoseregulated phosphorylation of TET 2 by AMPK reveals a pathway linking diabetes to cancer. Nature $\mathbf{5 5 9}$, 637-641 (2018)

FURTHER READING Li, X. et al. Regulation of chromatin and gene expression by metabolic enzymes and metabolites. Nat. Rev. Mol. Cell Biol. https://doi.org/10.1038/s41580-018-0029-7 (2018) 